IEKP-KA/2001-24

Nov. 2001

\title{
Proton and Photon Structure
}

\author{
Martin Erdmann \\ Institut für Experimentelle Kernphysik, Universität Karlsruhe, \\ Wolfgang-Gaede-Str. 1, D-76131 Karlsruhe \\ Martin.Erdmann@cern.ch
}

\begin{abstract}
The increasing precision of the measurements on the proton structure and an improved treatment of the correlated systematic experimental errors constitute a major step forward in our understanding of the flavour decomposition of the proton and the momentum distributions of the various flavours. Together with theoretical progress on the next-to-nextto-leading order QCD corrections to deep inelastic scattering processes, the proton measurements already imply a new level of precision for the strong coupling constant $\alpha_{s}$. The progress in the measurements on the quantum fluctuations of the photon allows questions on the universal properties of hadronic structures to be addressed.
\end{abstract}

\footnotetext{
${ }^{1}$ Invited talk at the XX International Symposium on Lepton and Photon Interactions at High Energies, Rome, Italy, July 2001
} 


\section{Introduction}

The research goals for the measurements of the proton and the photon structure are mainly twofold. One motivation is the physics understanding of hadronic structures concerning the momentum distributions of their partons and the quark flavours that contribute. This aspect is thoroughly analysed in different scattering processes with the proton, and in measurements of the hadronic structures developing from the quantum fluctuations of the photon (Fig. 1).
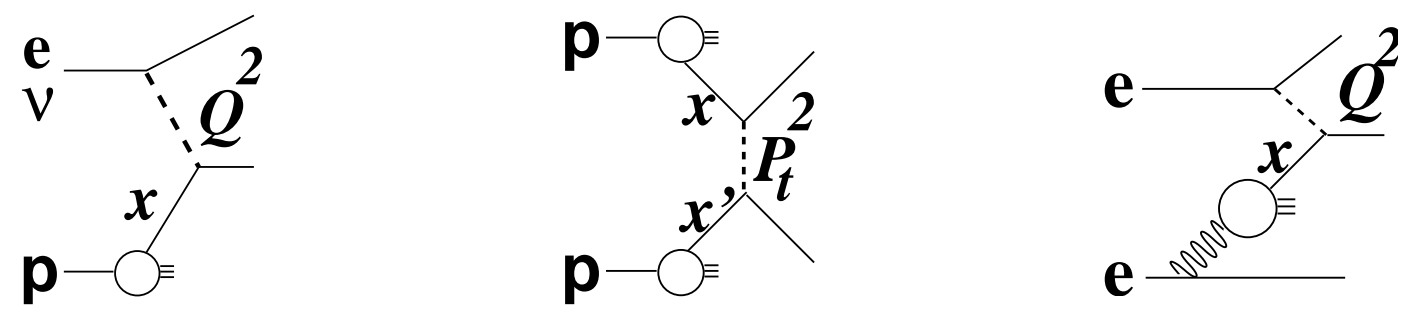

Figure 1: Examples of lepton-proton, proton-proton, and electron-photon scattering processes probing the proton and photon structure

The second aim is the test of the Standard Model in both the electroweak and the strong interaction sector. The new precision measurements of the proton structure function together with a new level of higher order QCD corrections results in most precise determinations of the strong coupling constant. Furthermore, unification of the electromagnetic and weak interactions is under direct observation by the recent deep inelastic scattering data. The distributions of observables related to the electroweak lepton-quark interactions are thoroughly scanned for possible new interactions and new heavy resonances.

\section{The Proton}

Two observables are required for the investigation of the proton structure: the Bjorken fractional momentum $x$ of the quark relative to the proton momentum (Fig. 1), and the resolution scale. In the case of deep inelastic lepton-proton scattering, the resolution scale is related to the negative squared four-momentum transfer $Q^{2}$, and for hadron-hadron scattering processes, it is related to the squared transverse momentum $P_{t}^{2}$ produced in the hard collision. In Fig. 2, the currently covered kinematic regions are shown, reaching in $x$ from $10^{-6}$ to almost 1 , and in $Q^{2}\left(P_{t}^{2}\right)$ from below 1 up to $10^{5} \mathrm{GeV}^{2}$. The latter values allow structures between $1 \mathrm{fm}$ down to $1 \mathrm{am}\left(10^{-18} \mathrm{~m}\right)$ to be resolved.

The new results are presented along a triangular walk through the kinematic plane. We start with the new HERA measurements at high $Q^{2}$, give the new results of the HERA high statistics region at lower $Q^{2}$, report on the new FNAL fixed target results in the same $Q^{2}$ region at higher values of $x$, and return to the high $Q^{2}$ regime with the new TEVATRON di-jet data. 


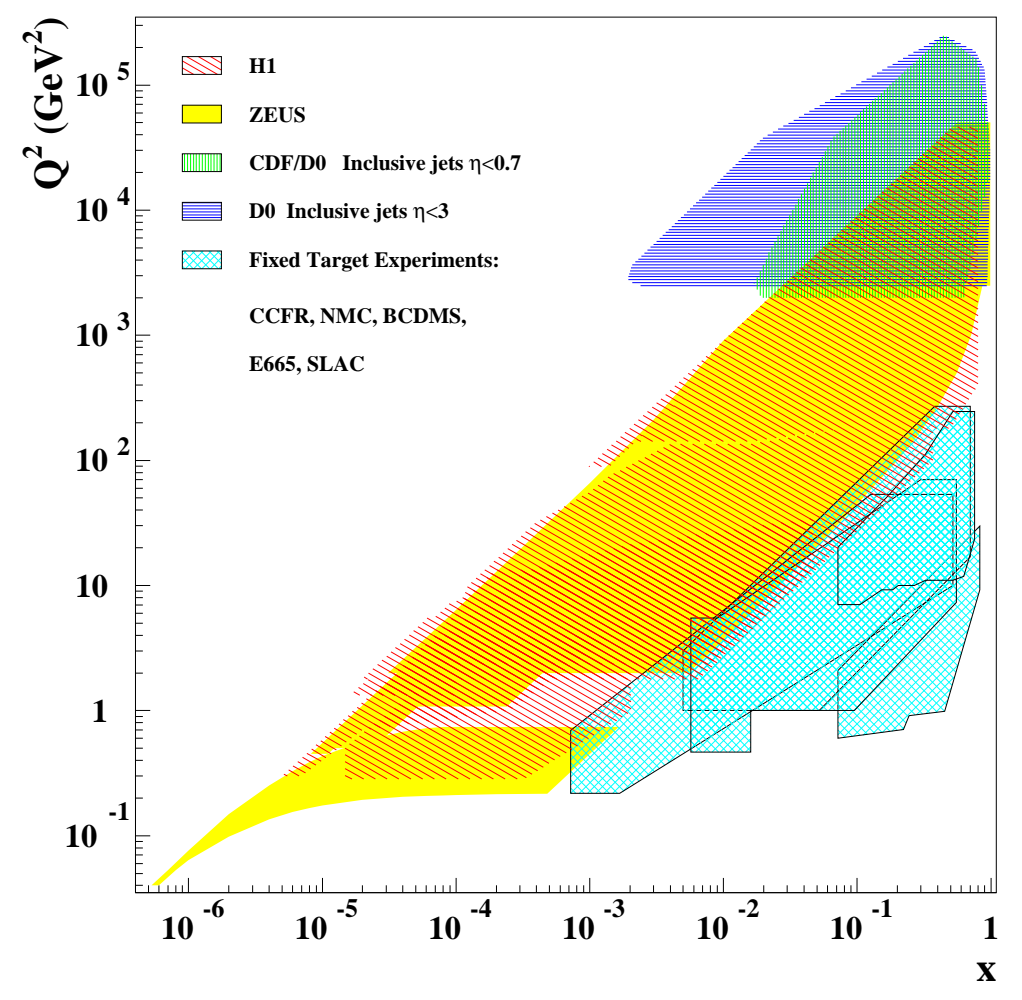

Figure 2: Kinematic plane

\subsection{Deep inelastic electron-proton scattering}

\subsubsection{Neutral and charged current cross sections}

The double differential cross sections for neutral and charged current lepton-proton scattering are given by eqs. (11) and (2).

$$
\begin{aligned}
& \frac{\mathrm{d}^{2} \sigma_{N C}}{\mathrm{~d} Q^{2} \mathrm{~d} x}=2 \pi \quad \alpha^{2} \quad \frac{1}{Q^{4}} \quad \frac{1}{x} \Phi_{N C}\left(x, Q^{2}\right) \\
& \frac{\mathrm{d}^{2} \sigma_{C C}}{\mathrm{~d} Q^{2} \mathrm{~d} x}=\frac{1}{2 \pi} G_{F}^{2}\left(\frac{M_{W}^{2}}{M_{W}^{2}+Q^{2}}\right)^{2} \frac{1}{x} \Phi_{C C}\left(x, Q^{2}\right)
\end{aligned}
$$

Here $\alpha$ and $G_{F}$ are the electromagnetic and Fermi coupling constants, respectively, $M_{W}$ denotes the $W$ boson mass, and the $\Phi$ terms contain the spin dependencies, the quark flavours, and, in the case of neutral current scattering, also the contributions of $Z$ boson exchange.

Equations (3-5) show approximate expressions for the $\Phi$ terms resulting from the helicity dependence $\left(y=Q^{2} / x / s_{e p}\right)$ of the lepton-quark interactions and the prevailing flavours at high $x$.

$$
\begin{aligned}
\Phi_{N C}^{l \pm N}\left(x, Q^{2}\right) & \sim\left(1+(1-y)^{2}\right)\left[\frac{4}{9} x u\left(x, Q^{2}\right)+\frac{1}{9} x d\left(x, Q^{2}\right)+\ldots\right] \\
\Phi_{C C}^{l+N}\left(x, Q^{2}\right) & \sim x \bar{u}\left(x, Q^{2}\right)+(1-y)^{2} x d\left(x, Q^{2}\right) \\
\Phi_{C C}^{l-N}\left(x, Q^{2}\right) & \sim x u\left(x, Q^{2}\right)+(1-y)^{2} x \bar{d}\left(x, Q^{2}\right)
\end{aligned}
$$




\subsubsection{Unification of the electroweak interactions}

The large center of mass energy of the HERA collider $\left(\sqrt{s_{e p}}=320 \mathrm{GeV}\right)$ allows the neutral and charged current interactions to be measured within a single experiment. In Fig. 3, measurements [1].2] of the neutral and charged current cross sections in positron-proton scattering are directly compared in terms of single differential cross sections as a function of $Q^{2}$.
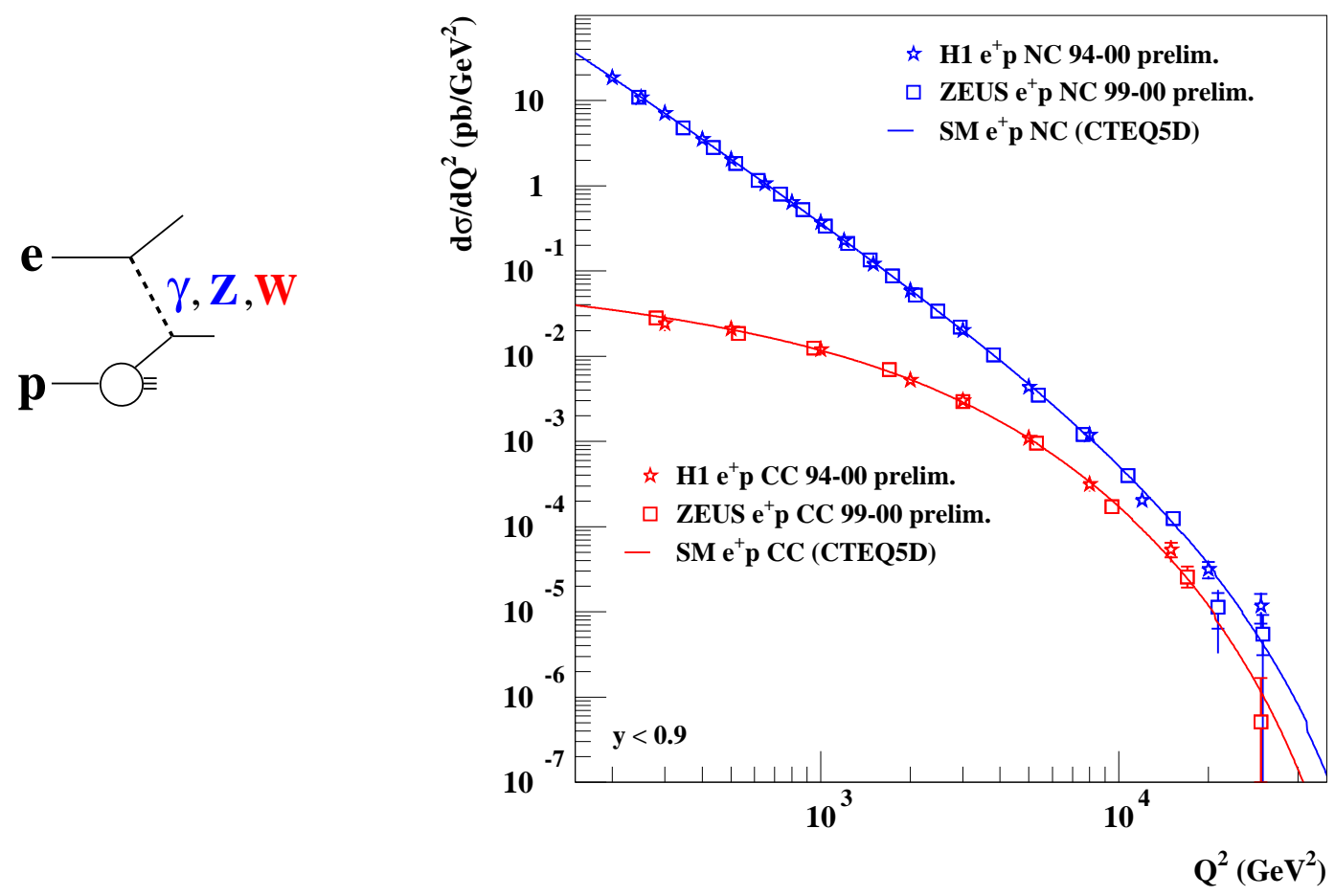

Figure 3: Neutral and charged current cross sections in positron-proton scattering

The neutral current data fall approximately according to $Q^{-4}$ as expected from photon exchange. The charged current measurements exhibit a weak $Q^{2}$ dependence at $Q^{2}$ values of a few hundred $\mathrm{GeV}^{2}$ due to the large $W$ mass. When $Q^{2}$ is of the order of the heavy boson masses squared, both cross sections are measured to be of similar magnitude.

This is a rather direct demonstration of the unification of the electromagnetic and weak interactions. Note that this is an approximate statement, since different quark flavours of the proton are probed in $e^{+} p$ scattering, the data are integrated over a large range of $x$, and the photon- $Z$ interference modifies the cross section for large $Q^{2}$.

\subsubsection{Helicity dependence and valence quarks}

Both HERA experiments have excellent measurements of the event kinematics which enables searches for new phenomena by scanning, e.g., the angular distributions of the lepton-quark $(e q)$ interactions in bins of the $e q$ center of mass energy. 

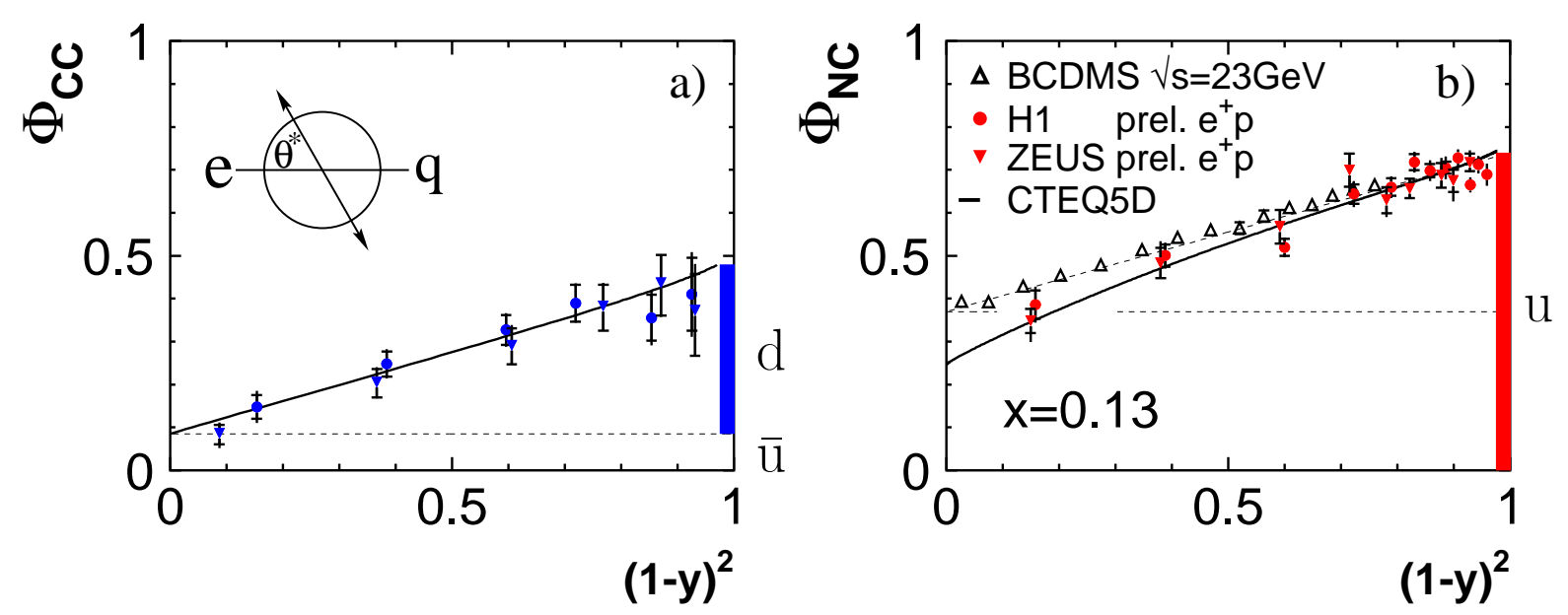

Figure 4: Helicity weighted parton distributions in charged and neutral current interactions

The helicity states of the lepton and the quark lead to two components in the distribution of the center of mass scattering angle $\theta^{*}$ (insert of Fig. Ta). If both incoming particles have the same handedness, any scattering angle is allowed and the cross section angular dependent weight of this component is unity. If the lepton and the quark have opposite handedness, the total spin of 1 cannot be flipped. Backward scattering is forbidden, and the cross section contribution is weighted by $\cos ^{4}\left(\theta^{*} / 2\right)=(1-y)^{2}$.

When analysing charged current interactions in positron-proton collisions, the positron couples only to negatively charged quarks, e.g. $d, \bar{u}$ (see eq. (円)). In addition, the right-handed positrons couple only to right-handed anti-quarks, and to left-handed quarks. In Fig. \#a, the $\Phi_{C C}$ term of eq. (2) is shown as a function of $(1-y)^{2} . \Phi_{C C}$ has been obtained by dividing the measured double differential cross section [1, 2] by the constant term of eq. (2), the Fermi coupling, the propagator term, and by multiplying by $x$ to get quark momentum distributions. Here $x=0.13$ has been chosen so as to minimise effects of gluon radiation processes. Within the errors of the measurements, the data are well compatible with a linearly rising distribution, showing the constant anti-quark contribution in the backward scattering region by the intercept $\left((1-y)^{2} \rightarrow 0\right)$. The quark contribution causes the rising component and is, at this value of $x$, mainly that of the $d$ valence quark.

In Fig. $4 \mathrm{~b}$, the corresponding neutral current measurement is shown. The open triangles show $\Phi_{N C}$ for the BCDMS data [3] at small center of mass energies $\left(\sqrt{s_{\mu p}}\right.$ up to $23 \mathrm{GeV}$ ). These data follow a linear rise where the intercept and the slope are of equal magnitude. This is expected for photon exchange (see eq. (3)). Owing to the charge dependence of the electromagnetic interactions, these data are mainly sensitive to the $u$ valence quark distribution at the given value of $x$. Both components of the angular distribution are weighted with $e_{u}^{2}=4 / 9$ such that in the forward scattering region $\left((1-y)^{2} \rightarrow 1\right)$ an approximate measure of the total $u$ valence quark density is obtained.

The HERA data, which probe the proton at much higher resolution scales, show the same $u$ quark density in the forward scattering region. The measurements in the backward scattering region $\left((1-y)^{2} \rightarrow 0\right)$ show the tendency to be below the BCDMS data which results from neg- 
ative interference between the photon and the $Z$ boson exchange in positron-proton scattering. The smaller cross section is confirmed in the neighbouring $x$-bins (not shown in the figure).

The direct comparison of the neutral current and charged current measurements reveals that the proton - also when probed with the high resolution scales at HERA - shows the familiar uud valence structure.

No significant sign of new physics has been observed in the inclusive measurements so far. An excess of the data over the Standard Model in the backward scattering region at $x \sim 0.4$, previously reported for the $e^{+} p$ data [4, 5], has not been confirmed with much larger integrated luminosity [6] and is hence attributed to a statistical fluctuation.

The HERA upgrade programme has already started and is expected to provide a factor of 10 more integrated luminosity than obtained so far. It will provide much higher precision for the flavour decomposition of the proton as well as for the consistency checks with respect to the Standard Model predictions.

\subsubsection{Measurements of the structure function $\boldsymbol{F}_{2}$}

In the region $1<Q^{2} \sim 100 \mathrm{GeV}^{2}$, each of the HERA experiments has several million events which are used to provide about 100 cross section measurements. In the high-statistics region, the accuracy is $2-3 \%$ which is dominated by systematic uncertainties [7, 8]. The measurements are expressed in terms of the electromagnetic proton structure function $F_{2}$ for photon exchange alone which is related to $\Phi_{N C}$ of eq. (1) in the way described below.

$\Phi_{N C}$ is commonly written in terms of the generalized structure functions $\tilde{F}_{2}, \tilde{F}_{L}, \tilde{F}_{3}$ shown in eq. (6). $\tilde{F}_{2}$ is related to the sum of the quark- and anti-quark distributions, and $\tilde{F}_{3}$ to their difference. $\tilde{F}_{L}$ describes the proton structure when probed by a longitudinal photon. $Y_{ \pm}=$ $1 \pm(1-y)^{2}$ give the helicity dependence of the lepton-quark scattering.

$F_{2}$ is contained in $\tilde{F}_{2}$ through eq. (7), where $F_{2}^{Z}$ and $F_{2}^{\gamma Z}$ are the contributions due to $Z$ exchange and $\gamma Z$ interference, respectively, $M_{Z}$ is the mass of the $Z$-boson,

$\kappa_{w}=1 /\left(4 \sin ^{2} \theta_{w} \cos ^{2} \theta_{w}\right)$ where $\theta_{w}$ is the Weinberg angle, and $v$ and $a$ are the vector and axial vector couplings of the electron to the $Z$. At small $Q^{2}$ and small $y$, the familiar relation (8) holds to a good approximation.

$$
\begin{aligned}
\Phi_{N C}^{l \pm N}\left(x, Q^{2}\right) & =Y_{+} \tilde{F}_{2}\left(x, Q^{2}\right)-y^{2} \tilde{F}_{L}\left(x, Q^{2}\right) \mp Y_{-} x \tilde{F}_{3}\left(x, Q^{2}\right) \\
\tilde{F}_{2} & =F_{2}-v \frac{\kappa_{w} Q^{2}}{\left(Q^{2}+M_{Z}^{2}\right)} F_{2}^{\gamma Z}+\left(v^{2}+a^{2}\right)\left(\frac{\kappa_{w} Q^{2}}{Q^{2}+M_{Z}^{2}}\right)^{2} F_{2}^{Z} \\
\Phi_{N C}\left(x, Q^{2}\right) & \sim\left(1+(1-y)^{2}\right) F_{2}\left(x, Q^{2}\right) \quad\left[\text { for } Q^{2}<1000 \mathrm{GeV}^{2}, y<0.3\right]
\end{aligned}
$$

In Fig. 5, the $F_{2}$ measurements are shown in bins of $x$ as a function of $Q^{2}$. The measurements are corrected for electroweak radiative effects. The data show strong scaling violations as expected from QCD radiative effects. 


\section{ZEUS+H1}

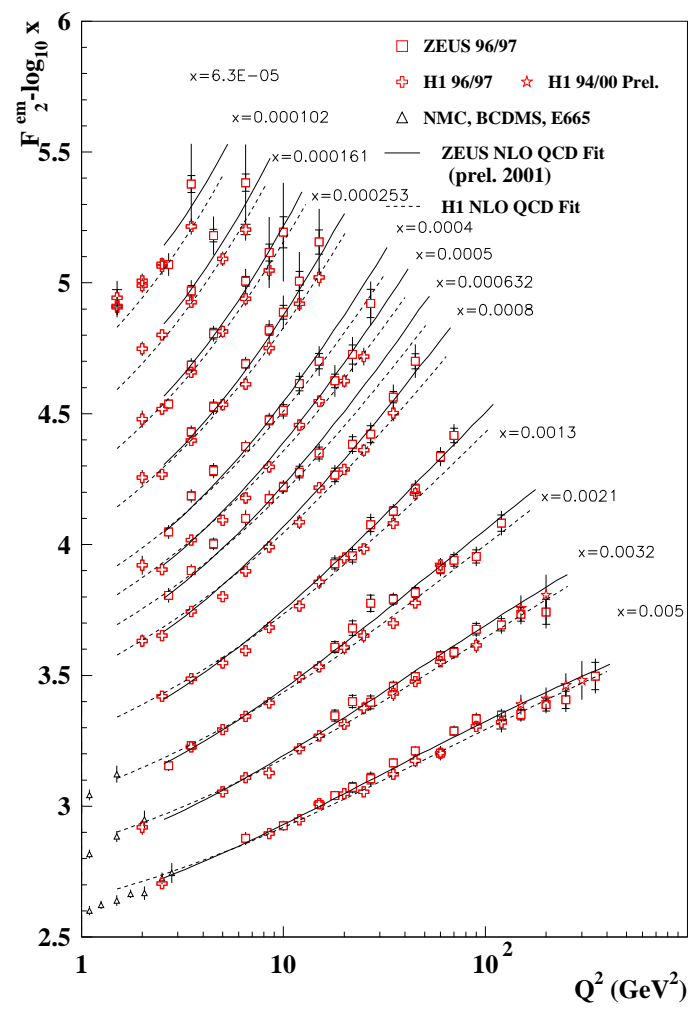

ZEUS+H1

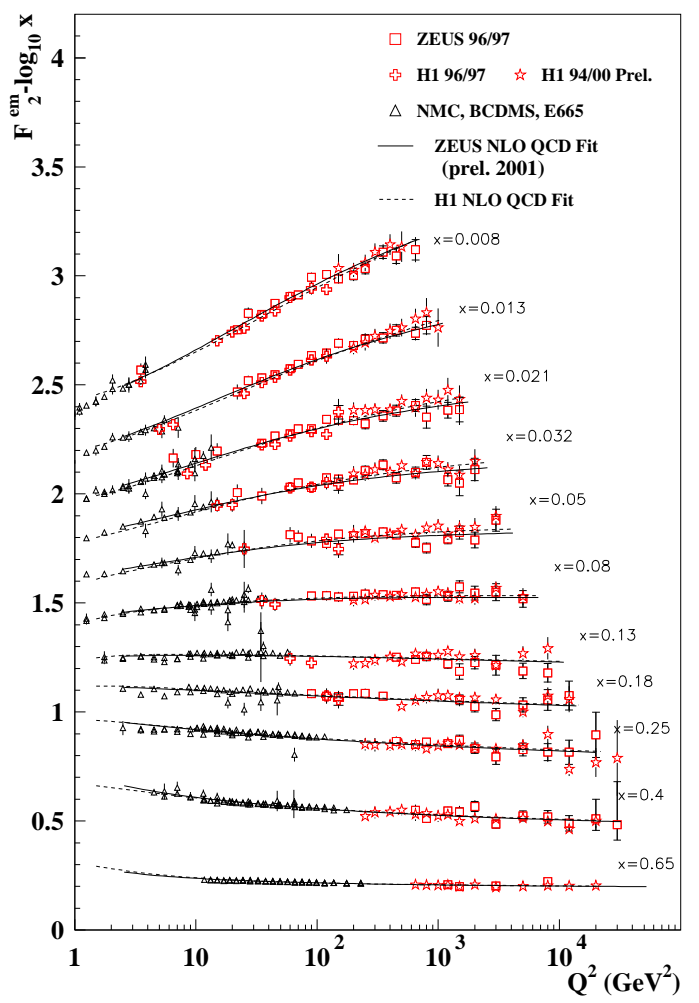

Figure 5: Proton structure function $F_{2}$

To provide an intuitive understanding of the hadronic structure as given by the data, we summarize the data at fixed $x$ values and for $2 \leq Q^{2} \leq 200 \mathrm{GeV}^{2}$ with two parameters $a(x)$ and $\kappa(x)$ [9]:

$$
F_{2}\left(x, Q^{2}\right)=a(x)\left[\ln \left(\frac{Q^{2}}{\Lambda^{2}}\right)\right]^{\kappa(x)} .
$$

The scale $\Lambda$ is chosen to be a typical value of the strong interaction scale $\Lambda=350 \mathrm{MeV}$. The parameter $\kappa$ reflects the strength of the scaling violations, and $a$ represents the quark distribution at $Q^{2}=0.3 \mathrm{GeV}^{2}$. Here the logarithmic term of $(9)$ is 1 and therefore $F_{2}$ is independent of $\kappa$. The data are well described by the fits using eq. (9) (not shown here).

The fit results for the parameter $a$ are presented in Fig. 6. The BCDMS data show the valence quarks around the value $x \sim 1 / 3$. The HERA data access the low- $x$ region and reveal a sea quark distribution which is consistent with being constant in $x$.

Around $x=0.1$, the parameter $\kappa$ is zero such that $F_{2}$ scales. Large negative scaling violations are visible above $x=0.1$, and positive scaling violations below that value which appear to rise towards small $x$. With the current data precision it remains an open question whether or not the scaling violations keep rising towards small $x$ which may give information on upper bounds for parton densities.

Some differences in the strength of the scaling violations $\kappa(x)$ between the measurements of the H1 and ZEUS experiments are apparent in $a(x)$ which need to be clarified. 


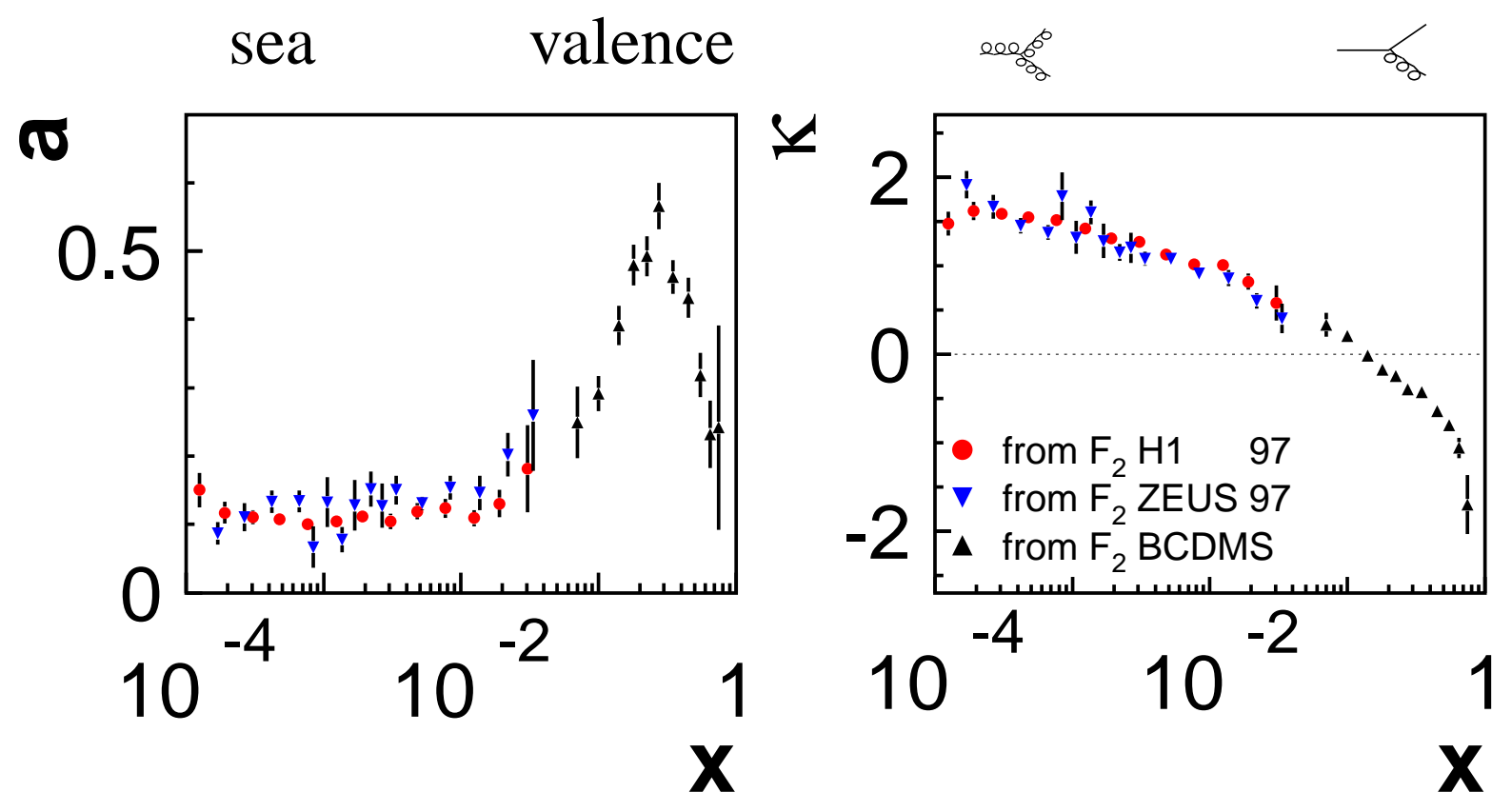

Figure 6: Quark distribution $a(x)$ of the proton at $Q^{2}=0.3 \mathrm{GeV}^{2}$ and scaling violations $\kappa(x)$

The QCD radiative effects causing the scaling violations give access to the gluon distribution and the strong coupling constant $\alpha_{s}$. Since the splitting functions dominating the radiative effects depend on the region of $x$ (see examples of the splitting functions at the top of Fig. (6), the gluon distribution and $\alpha_{s}$ can be determined simultaneously.

In Fig. 7 7 a, the gluon distribution of the proton is shown for $Q^{2}=5 \mathrm{GeV}^{2}$ from the H1 experiment [7]. In the $x$ regions where the $Q^{2}$ lever arm is longest, $10^{-3}<x<10^{-2}$, the precision is of order $10 \%$. The gluon distribution as determined from the ZEUS experiment [10] is shown in Fig. 7 $7 \mathrm{~b}$ together with the other parton distributions extracted from the data at $Q^{2}=10 \mathrm{GeV}^{2}$.

\subsubsection{Determination of the strong coupling constant $\alpha_{s}$}

In Fig. 8, determinations of the strong coupling constant $\alpha_{s}$ are shown, grouped in NLO and NNLO QCD extractions. As a reference, the grey band shows the value given in a recent review of Bethke [11]. Both HERA experiments [7, 10] extract $\alpha_{s}$ at the NLO QCD level. Their $\alpha_{s}$ values are fully compatible with each other. The $\mathrm{H} 1$ experiment used their own data and fixed target data from proton target only, avoiding thereby target mass corrections and regions affected by higher twist effects. Their total experimental error is 0.002 , shown by the second symbol. The total error bar is dominated by the renormalisation scale uncertainty. In this analysis, the scale was varied by a factor 2 down and up.

These theoretical uncertainties are expected to be much smaller when using higher order calculations. The next-to-next-to leading order (NNLO) corrections are partly available [12]. The two-loop coefficient functions of $F_{2}, F_{3}$ and $F_{L}$ have been calculated [13], and have been 
a)

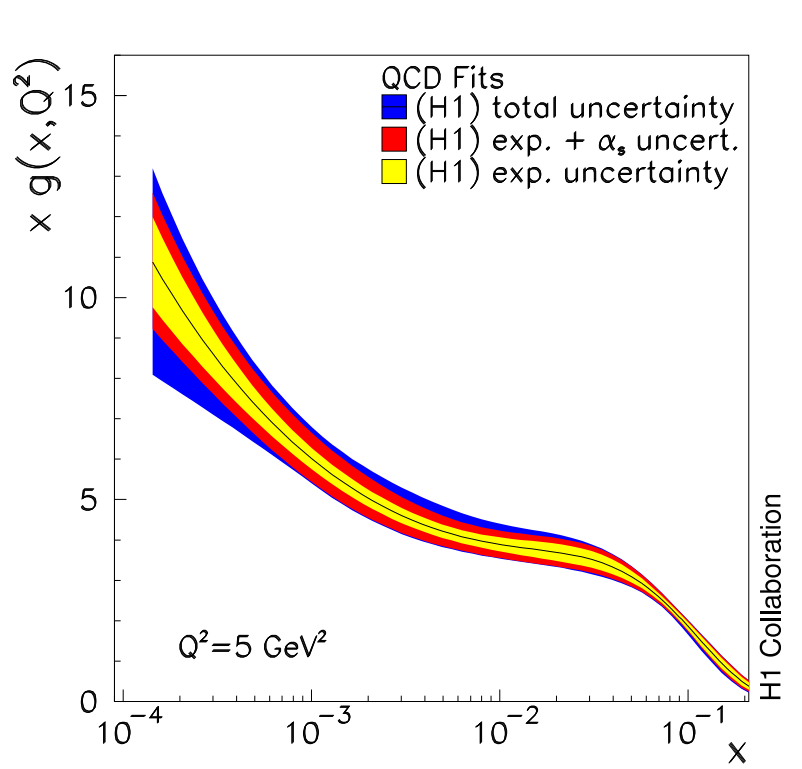

b)

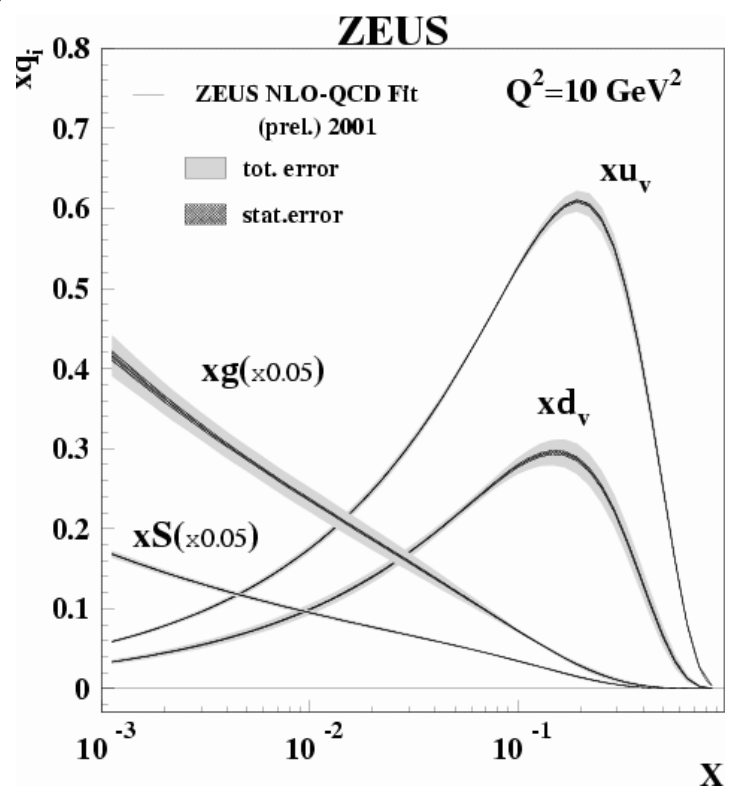

Figure 7: a) Gluon distribution of the proton from $\mathrm{H1}$, b) parton distributions of the proton from ZEUS

completely checked [14]. For the three-loop anomalous dimensions $\gamma_{\mathrm{pp}}^{(2)}$, only partial results are available thus far. These include a finite number of fixed Mellin moments [15, 16], both for $F_{2}$ and $F_{3}$, the large $n_{f}$-limit [17] of $\gamma_{\mathrm{qq}}^{(2)}$ and $\gamma_{\mathrm{gg}}^{(2)}$, in the latter case only the coefficient of the colour factor $n_{f}^{2} C_{A}$, and several terms relevant to the small- $x$ limit [18].

The corrections available so far are already sufficient to determine $\alpha_{s}$ at the NNLO QCD level by averaging the data in $Q^{2}$ bins to calculate Mellin moments. This approach has been followed by Santiago and Yndurain where the results [19] are shown in Fig. 8. The error from the extraction using $e p$ and $\mu p$ data is extremely competitive when compared to other determinations of $\alpha_{s}$. The central value and the experimental uncertainty should be confirmed by other groups. Regarding the theoretical uncertainties in $\alpha_{s}$ from deep inelastic scattering, van Neerven and Vogt also expect a theoretical error below 1\% [20]. Determinations of $\alpha_{s}$ [19, 21] using $\nu N$ data agree within errors which are, however, larger compared to the $e p, \mu p$ case (Fig. 8).

It can be anticipated that the theoretical progress in the NNLO QCD corrections together with the increasing precision in the $F_{2}$ measurements will lead to the most precise determinations of $\alpha_{s}$.

\subsection{Deep inelastic neutrino-nucleon scattering}

The neutrino data cover a complementary kinematic region up to $Q^{2}=250 \mathrm{GeV}^{2}$ and $0.01<$ $x<1$. These data are sensitive to the different quark flavours and are dominated by charged current interactions. 


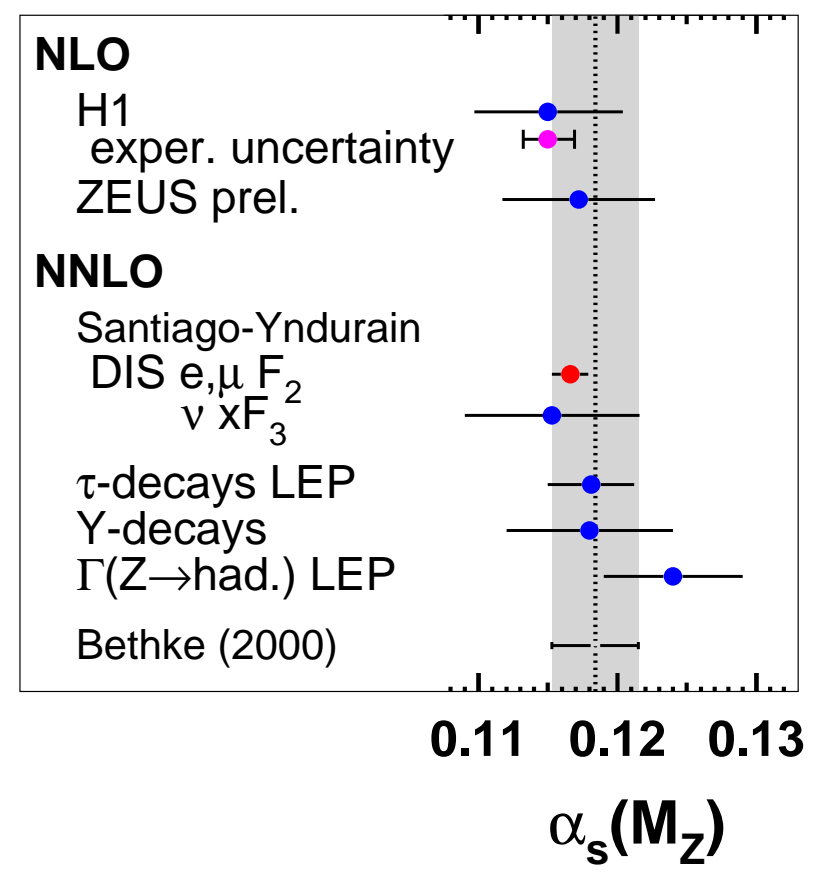

Figure 8: Determinations of the strong coupling constant

Recently the CCFR collaboration has re-analysed their data [22] in the light of major improvements in the theoretical understanding of the heavy quark contributions. They changed the analysis method of extracting information on the structure functions. Their $F_{2}$ data are now well consistent with the charged lepton data at the level of $5 \%$, after correcting for the charge factor 18/5, and when using NLO QCD calculations of the heavy quark contributions.

The successor experiment [23] NUTEV has overall much better experimental conditions. The collaboration just released a first glance at their $F_{2}$ data so far with statistical errors only (Fig. 9). While in this measurement, the data sets of the neutrino and anti-neutrino beams have been combined, the clean separation of the $\nu$ and $\bar{\nu}$ beams give them the potential for extracting momentum distributions for different quark flavours in the proton.

\subsection{Drell-Yan production of muon pairs}

Measurements of the Drell-Yan production of muon pairs give access to the anti-quark distributions of the proton at high values of $x$ [24]. The ratio of proton-deuterium and proton-hydrogen target data is directly related to the $\bar{d} / \bar{u}$ ratio. For highly asymmetric kinematics of the fractional momenta of the incoming quark and anti-quark, $x_{1} \gg x_{2}$, both of which can be calculated from the kinematics of the muon pair, the ratio is given by

$$
\frac{\sigma(p d)}{2 \sigma(p p)} \sim \frac{1}{2}\left[1+\frac{\bar{d}\left(x_{2}\right)}{\bar{u}\left(x_{2}\right)}\right] .
$$

This relation is valid for the assumption that the parton distributions of the nucleon obey charge symmetry, $u_{p}(x)=d_{n}(x)$ etc.

Fig. 10 gives the measured ratio $\bar{d} / \bar{u}$ as a function of $x$ showing a large asymmetry which is likely to be of non-perturbative origin. 


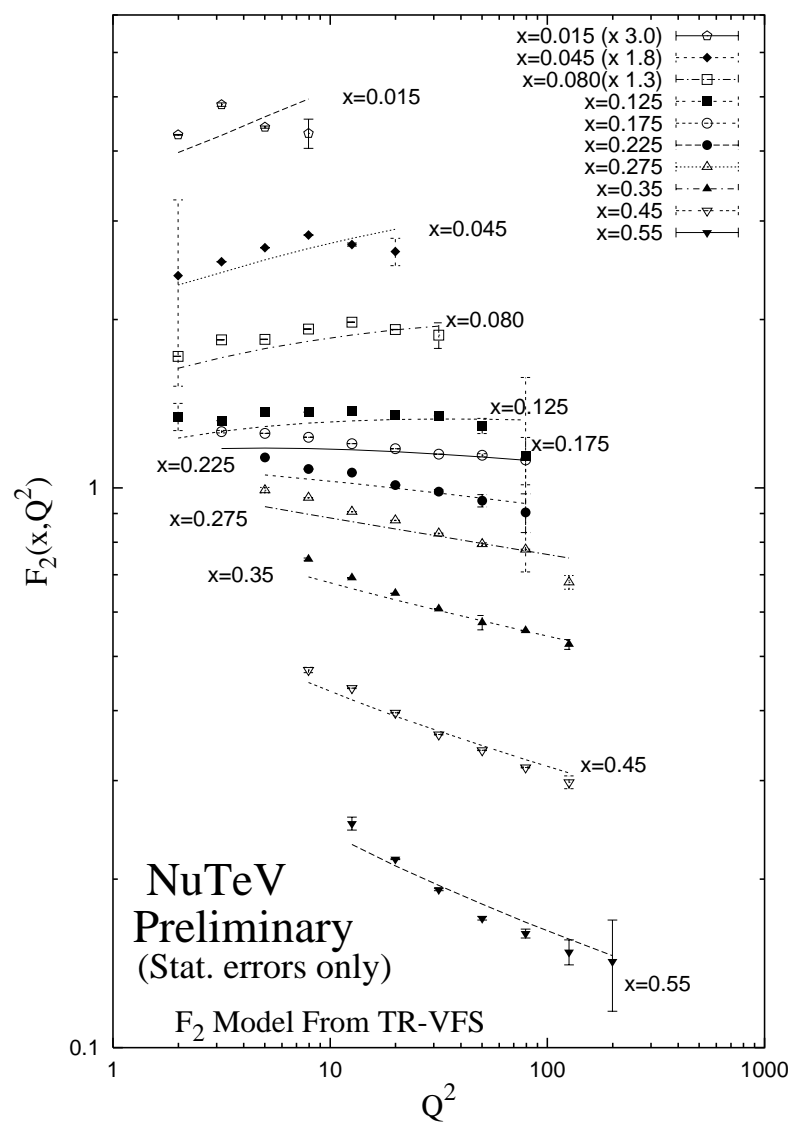

Figure 9: Proton structure function $F_{2}$ from neutrino-nucleon scattering
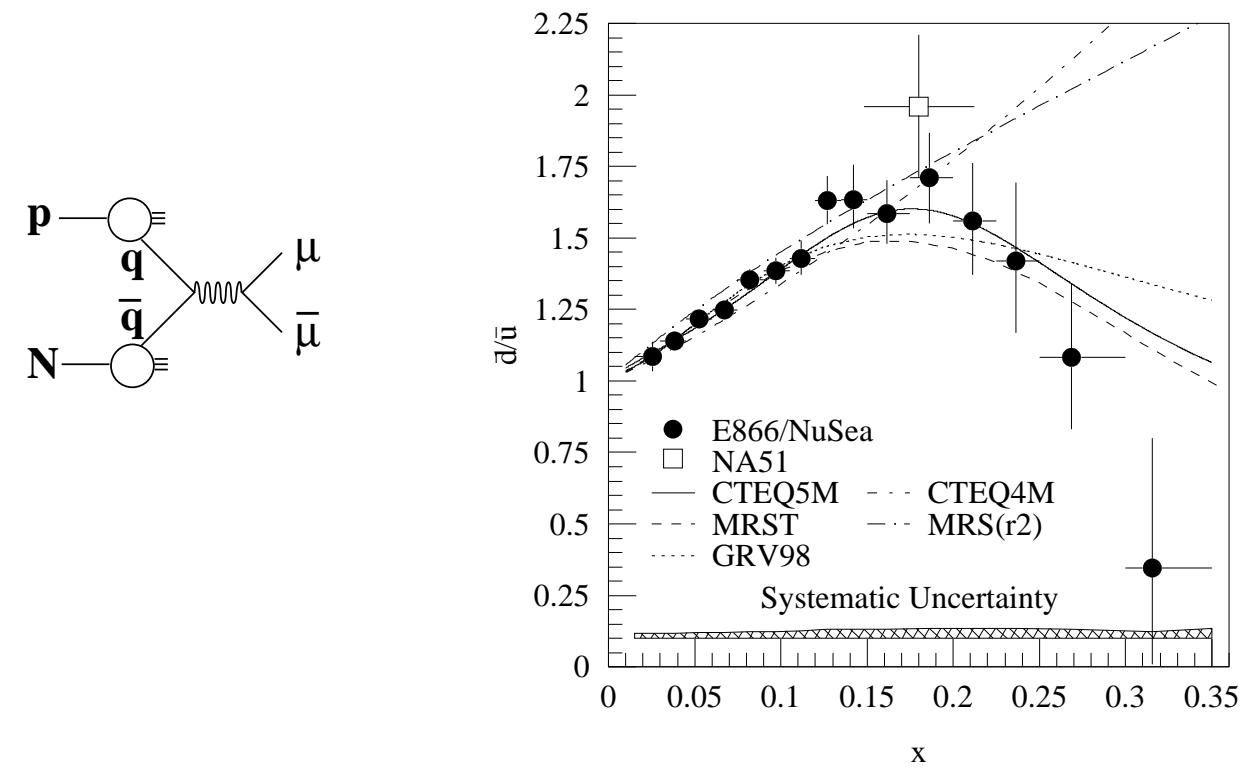

Figure 10: Ratio of the anti-down- over anti-up-quark distributions in the proton 


\subsection{Di-jet cross sections in hadron-hadron collisions}

Jet measurements at the TEVATRON collider challenge the Standard Model at the smallest distance scales and simultaneously constrain the parton distributions of the proton. Both collaborations CDF [25] and D0 [26] have published measurements of the di-jet cross sections in terms of the jet transverse energies $E_{T, i}$ and their pseudo-rapidities $\eta_{i}(i=1,2)$ (Fig. 11).
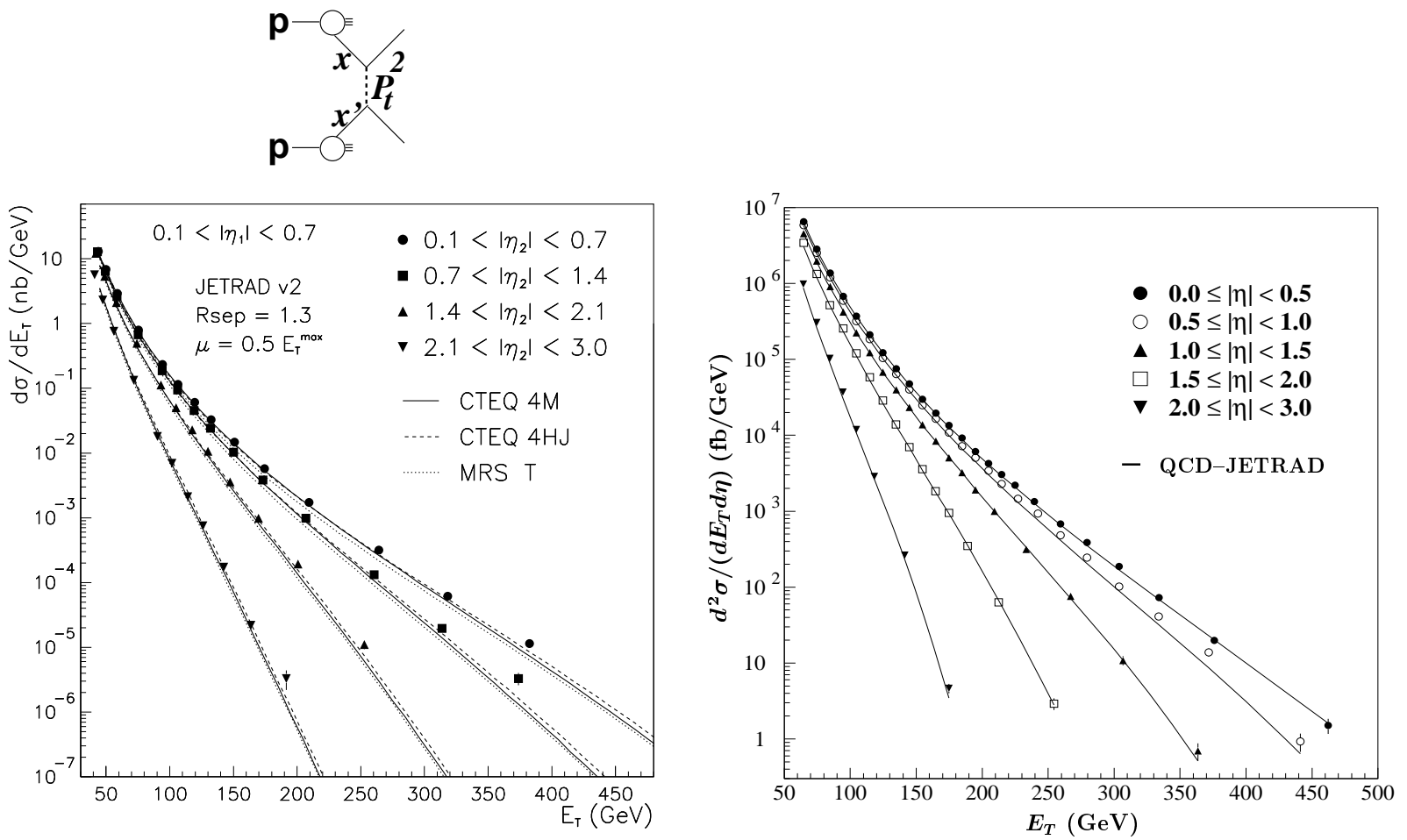

Figure 11: Differential di-jet cross section in proton-anti-proton collisions from the CDF and D0 experiments

In leading order QCD, these observables are related to the parton fractional momenta $x_{ \pm}$of the two incoming partons via

$$
x_{ \pm}=\frac{E_{T}}{\sqrt{s_{p \bar{p}}}}\left(\exp \left( \pm \eta_{1}\right)+\exp \left( \pm \eta_{2}\right)\right) .
$$

The data give access to the colour charge weighted sum of the quark and gluon distributions in the proton. They cover the high- $Q^{2}-$ high- $x$ region of Fig. 2. Here the quark distributions are rather well constrained so that the di-jet data give information on the high- $x$ gluon distribution of the proton.

The experiments express the sensitivity of their data by comparing with NLO QCD calculations using different sets of parton distributions of the proton obtained from global fits. While the D0 collaboration reports a consistent description of their measurements with some of the sets, the CDF collaboration finds that no set describes their data. It is planned to include the di-jet data in global fits of the parton distribution functions. This enables studies of the high- $x$ gluon distribution of the proton, and of the differences in the data of the two experiments. 


\subsubsection{How good are predictions for cross sections involving proton structure}

A frequently asked question is, how well do we know the parton distributions of the proton? Recently, major progress has been achieved concerning the correct treatment of the correlated experimental errors in global fits [7, 10,27-30]. These developments provide the tools to combine different data sets with a clear interpretation of the resulting $\chi^{2}$ per degree of freedom, and allow error bands to be drawn for the parton distributions. Examples are shown in Fig. ㄱ.

To understand the impact of our knowledge of the proton structure on cross section predictions, e.g. for the LHC, benchmark processes are being studied and are directly related to the proton structure function measurements. For example, the $W$-boson production cross section is varied with respect to the incoming partons. It is then checked whether these variations are within the uncertainties of the structure function data. Recent studies [31, 32] indicate an accuracy of the $W$ production cross section at the LHC of $2-4 \%$.

\section{The Photon}

Quantum fluctuations of the photon provide an ideal laboratory for analysing the genesis process of hadronic structures. The splitting of the photon into a quark-anti-quark pair at quark fractional momenta $0.1<x<1$ was already rather well understood from comparisons of the measurements at the PETRA and PEP $e^{+} e^{-}$colliders with the theoretical predictions. In contrast, the gluon developing in the hadronic fluctuations and the quark structure at low $x$ were essentially unknown before HERA and LEP.

\subsection{Deep inelastic electron-photon scattering}

In Fig. 12, a diagram of deep inelastic electron-photon scattering is shown. As for the proton case, the double differential cross section is related to the photon structure function $F_{2}^{\gamma}$ by eqs. (1.,8). The contributions of the structure function $F_{L}$ describing the structure of the transverse photon when probed by a longitudinal photon, and the contributions of the $Z$ boson exchange are small in the kinematic region presently covered and are usually neglected in the analysis.

In Fig. 12, measurements [33-35] of $F_{2}^{\gamma} / \alpha$ are shown as a function of $x$ for $3.7<\left\langle Q^{2}\right\rangle<5$ $\mathrm{GeV}^{2}$ (data comparison as in [36]). In the past few years, major improvements in the analysis techniques, especially at low- $x$, have been achieved, giving an accuracy of $F_{2}^{\gamma}$ of the order of $10 \%$. The curves represent the predictions of Glück, Reya, and Schienbein for the purely perturbative contribution, and separately the summed perturbative and non-perturbative parts [37]. The deviation of the data from the perturbative part indicates the hadron-like contribution to the structure function data.

In Fig. 13, measurements of $F_{2}^{\gamma} / \alpha$ are summarized as a function of $Q^{2}$ in bins of $x$ (updated version from [38]). In comparison to the previous measurements of the photon structure, the LEP data access the high- $Q^{2}$ region up to $10^{3} \mathrm{GeV}^{2}$, and the low- $x$ regime down to $x \sim 10^{-3}$ for $Q^{2} \geq 2 \mathrm{GeV}^{2}$. 


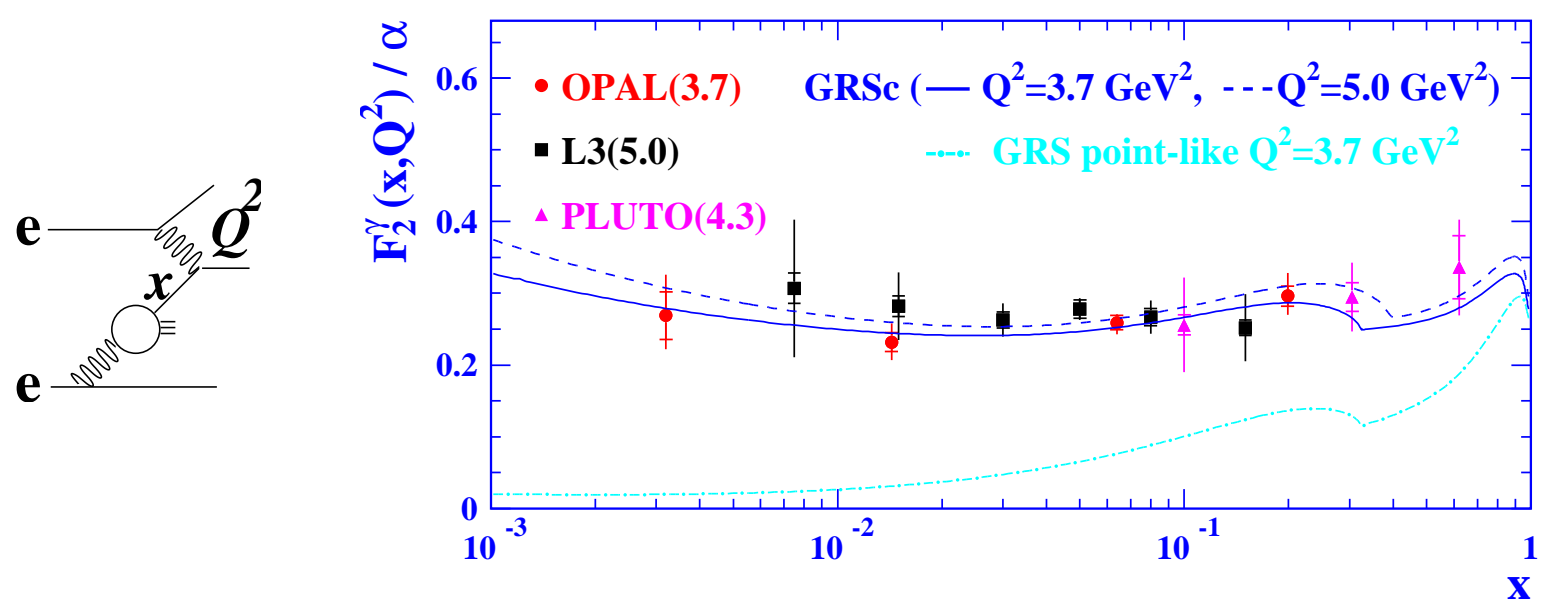

Figure 12: Photon structure function $F_{2}^{\gamma} / \alpha$ at low- $x$

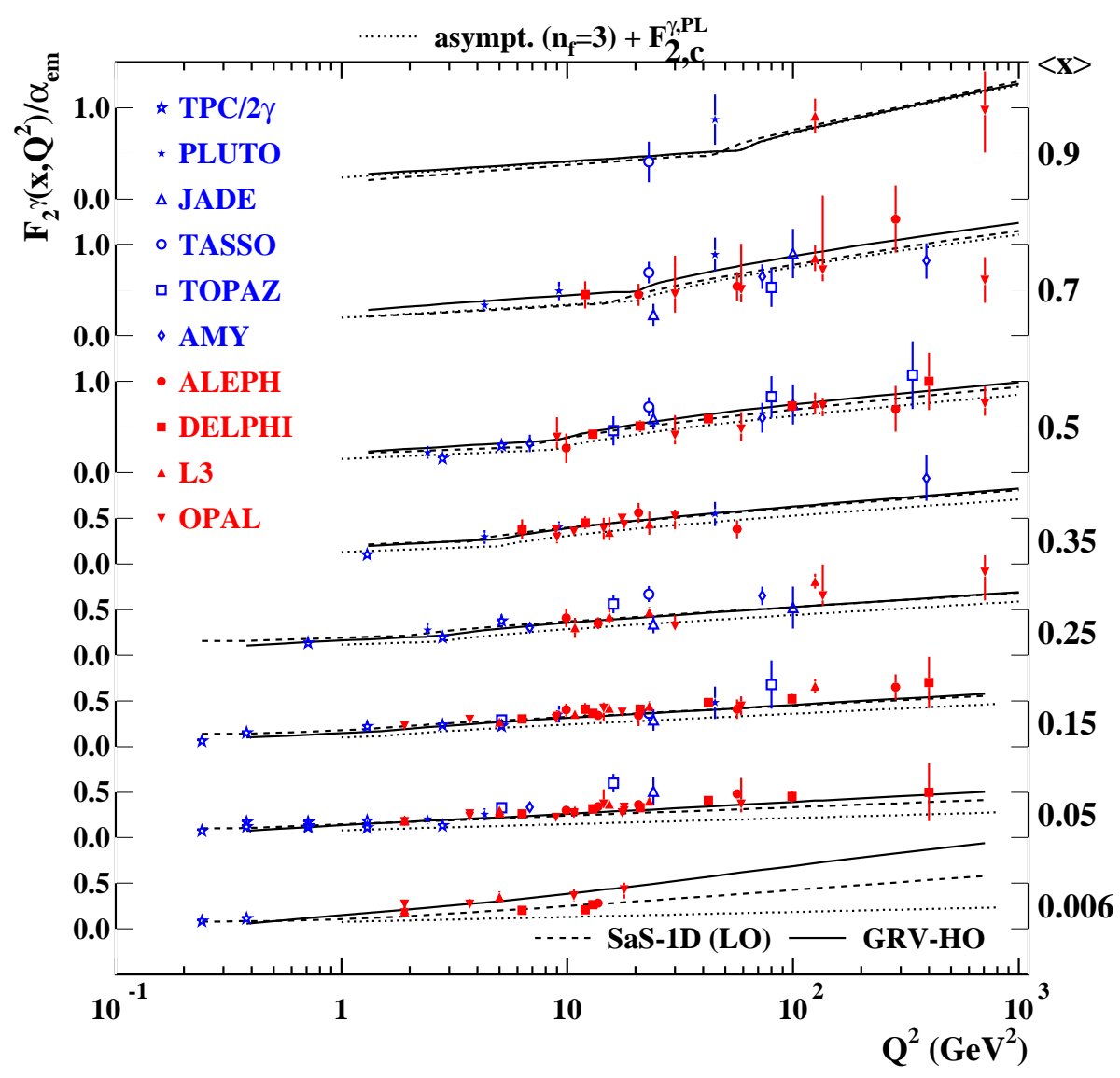

Figure 13: Photon structure function $F_{2}^{\gamma} / \alpha$

It is instructive to compare the photon data to the hadronic structure of the proton. In Fig. 14, the measurements of $F_{2}^{\gamma} / \alpha$ are analysed using the same description eq. (9) as used above for the proton. The photon shows no valence quark structure, as expected for this quantum fluctuation. 
It is interesting to note that the values of the parameter $a$, that describes the quark distribution for $Q^{2}=0.3 \mathrm{GeV}^{2}$, are at the same level as those of the sea quarks in the proton. At large values of $x>0.1$, the scaling violations $\kappa$ are expected to be of order 1 from the splitting of the photon into a quark-anti-quark pair [39]. The data are fully compatible with this prediction. They strongly differ from the scaling violations observed in the measurements of the proton structure.

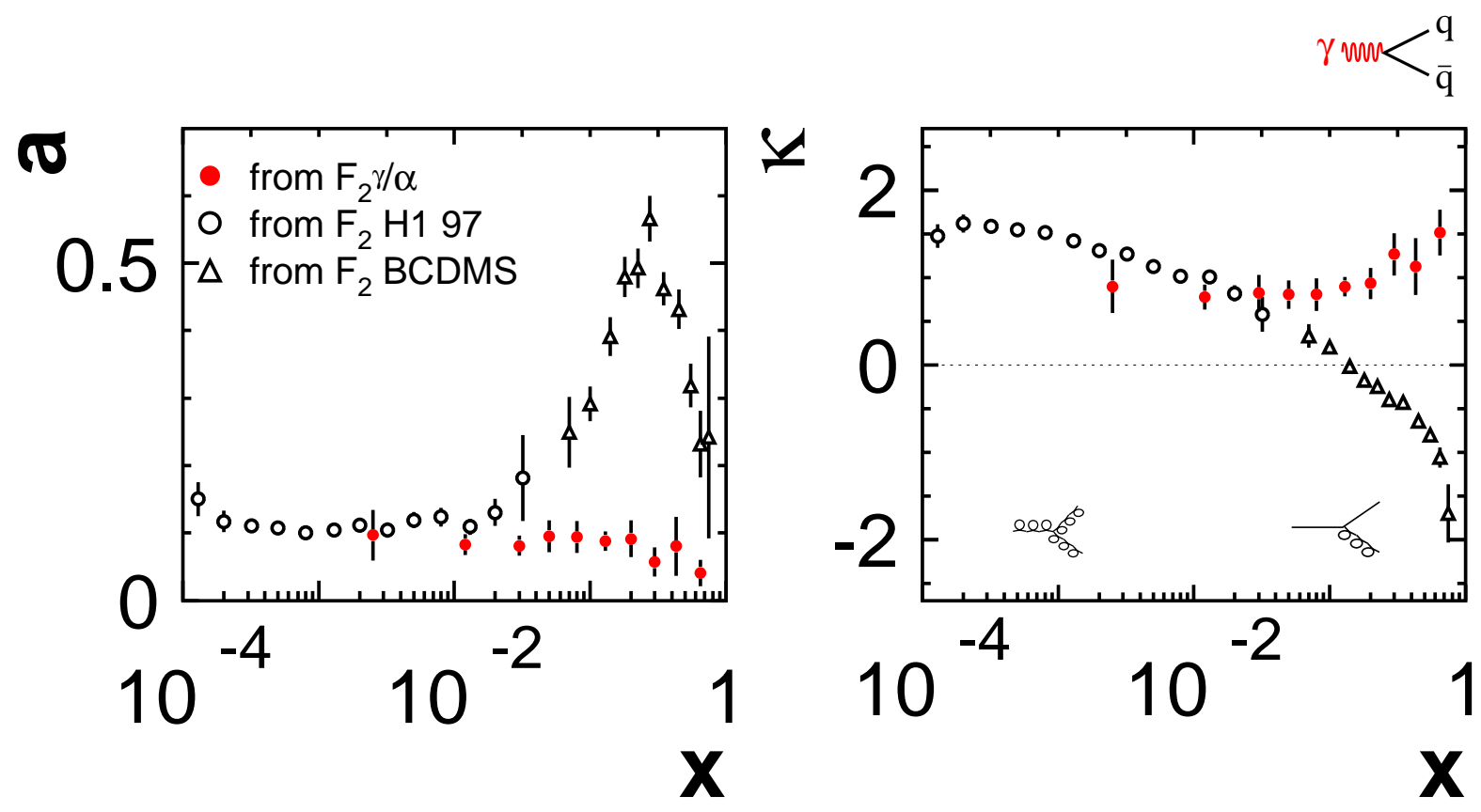

Figure 14: Quark distribution of the photon at $Q^{2}=0.3 \mathrm{GeV}^{2}$ and corresponding scaling violations (closed symbols) compared to the proton (open symbols)

A particular virtue of the photon measurements is their potential to answer questions on universal properties of hadronic structures, e.g., do parton radiation processes lead to universal hadronic structures at small values of $x$ ? At low values of $x$, the proton data exhibit positive and increasing scaling violations from the contribution of the gluons. The photon data now access this interesting domain of phase space; however, the precision of the data is not yet good enough to decide whether or not the scaling violations start to deviate from 1 and follow the scaling violations of the proton.

\subsection{Di-jet measurements from photon-proton and photon-photon colli- sions}

While the deviation of the data from the purely perturbative prediction in Fig.12 gives indirect evidence for a gluon contribution to the hadronic structure, the first direct measurement of the gluon distribution of the photon has been obtained in di-jet measurements at HERA [40]. In Fig. [15a, a recent measurement from $\mathrm{H} 1$ [41] is shown in comparison to the gluon as measured in the proton [7]. The data are compared at the resolution scales $Q^{2}=p_{t}^{2} \sim 70 \mathrm{GeV}^{2}$. Although 
the photoproduction measurement is limited in precision owing to underlying event effects, the similarity of the distributions possibly hints to a universal gluon distribution in hadronic structures which are based on quarks.

a)
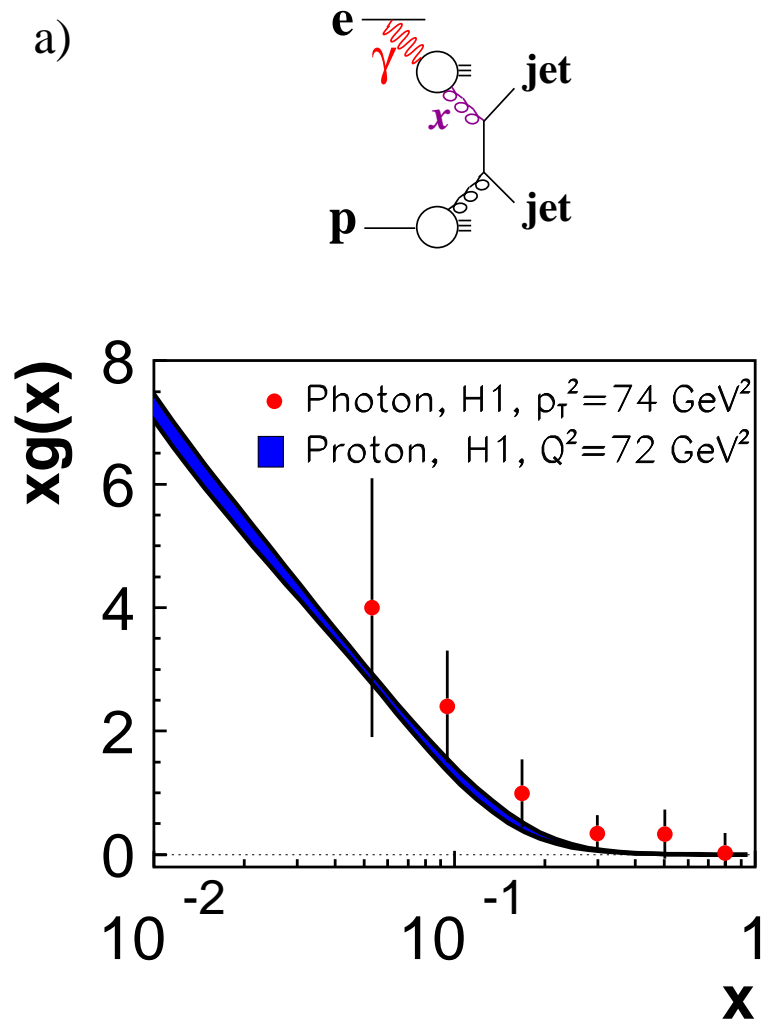

b)

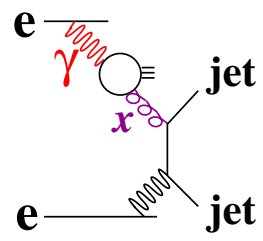

OPAL Preliminary

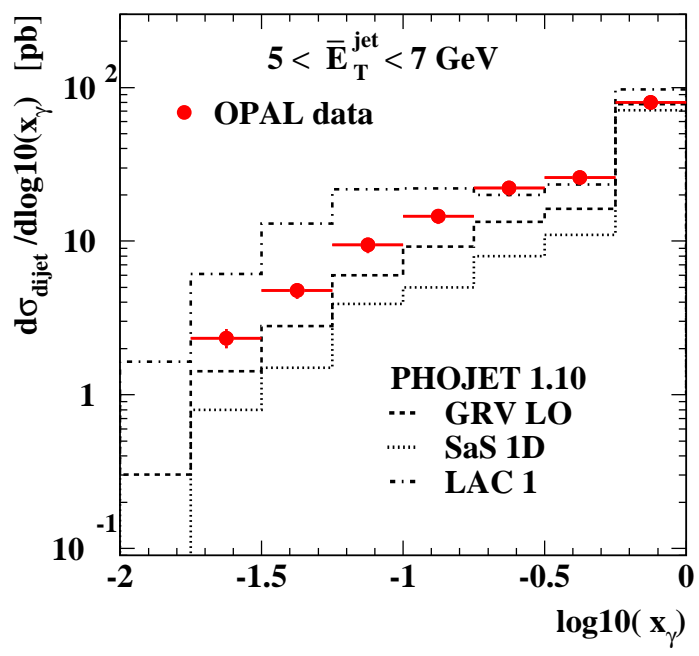

Figure 15: a) Gluon distribution of the photon from di-jet measurements in $\gamma p$ interactions in comparison to the gluon distribution of the proton, b) di-jet cross section in $\gamma \gamma$ interactions compared to QCD calculations using different input parton distributions for the photon

Major improvements in the precision of the gluon distribution of the photon are expected to come from LEP data where effects of the underlying event can be avoided, in principle. In Fig. 15b, a preliminary OPAL measurement [42] of the differential di-jet cross section is shown as a function of the logarithm of $x$. The different histograms represent calculations of the cross section using different input parton distributions, and demonstrate the high sensitivity of the data to the gluon contribution.

\section{Concluding remarks}

Structure function measurements again become a field of precision data, this time covering a much larger phase space in $x$ and $Q^{2}$. Together with new theoretical developments on NNLO QCD corrections (and beyond) to deep inelastic lepton-proton scattering, the data already imply a new level of precision for the value of the strong coupling constant. 
Comparisons of the proton data with measurements on the photon structure allow universal properties of hadronic structures to be studied directly from the measurements themselves. The recent photon data cover the relevant phase space and are in precision close to what is needed for firm statements.

\section{Acknowledgments}

For discussions and kind help, I wish to thank many members of the CERN, DESY, and FNAL experiments. I am especially grateful to I. Bertram, S. Bethke, A. Cooper, A. Dubak, E. Elsen, F. Happacher, M. Klein, M. Moritz, D. Naples, C. Niebuhr, R. Nisius, E. Rizvi, P. Schleper, S. Söldner-Rembold, M. Stanitzki, W. Tung, P. Valente, R. Wallny, T. Wengler, J. Whitmore, H. Wieber, R. Yoshida, and the Rome Team! I wish to thank S. Moch for his kind advice concerning the NNLO developments in deep inelastic scattering. I wish to further thank Th. Müller and the IEKP group of the University Karlsruhe for their hospitality, and the Deutsche Forschungsgemeinschaft for the Heisenberg Fellowship.

\section{References}

[1] H1 Collab., "Inclusive Measurement of Deep Inelastic Scattering at high $Q^{2}$ in ep Collisions at HERA", abs. 787, 803, Intern. Europhys. Conf. on High Energy Physics (2001), Budapest, Hungary; abs. 481, 496, XX Intern. Symp. on Lepton and Photon Interactions at High Energies (2001), Rome, Italy

[2] ZEUS Collab., "Measurement of high- $Q^{2}$ neutral current cross sections in $e^{+} p$ deep inelastic scattering at HERA", and "Measurement of high- $Q^{2}$ charged current cross sections in $e^{+} p$ deep inelastic scattering at HERA", abs. 630, 631, Intern. Europhys. Conf. on High Energy Physics (2001), Budapest, Hungary

[3] BCDMS Collab., Benvenuti et al., Phys. Lett. B 223 (1989) 485

[4] H1 Collab., C. Adloff et al., Z. Phys. C 74 (1997) 191

[5] ZEUS Collaboration; J.Breitweg et al., Z. Phys. C 74 (1997) 207

[6] H1 Collab., "A Search for Leptoquark Bosons in ep Collisions at HERA", abs. 822, Intern. Europhys. Conf. on High Energy Physics (2001), Budapest, Hungary abs. 514, XX Intern. Symp. on Lepton and Photon Interactions at High Energies (2001), Rome, Italy

[7] H1 Collab., C. Adloff et al., Eur. Phys. J. C 21 (2001) 33

[8] ZEUS Collab., S. Chekanov et al., Eur. Phys. J. C 21 (2001) 443

[9] M. Erdmann, Phys. Lett. B 488 (2000) 131

[10] ZEUS Collab., "NLO-QCD fit to determine proton PDFs and $\alpha_{s}$ ", abs. 628, Intern. Europhys. Conf. on High Energy Physics (2001), Budapest, Hungary 
[11] S. Bethke, J. Phys. G 26 (2000) R27

[12] S. Moch, J.A.M. Vermaseren, M. Zhou "Developments in Deep-inelastic Structure Function Calculations", Proc. of Intern. Europhys. Conf. on High Energy Physics (2001), Budapest, Hungary, hep-ph/0108033

[13] E. B. Zijlstra and W. L. van Neerven, Phys. Lett. B 272 (1991) 127; 273 (1991) 476; 297 (1992) 377; Nucl. Phys. B 383 (1992) 525

[14] S. Moch and J. A. M. Vermaseren, Nucl. Phys. B 573 (2000) 853; Nucl. Phys. Proc. Suppl. 86 (2000) 78; 89 (2000) 137

[15] S. A. Larin, P. Nogueira, T. van Ritbergen, and J. A. M. Vermaseren, Nucl. Phys. B 492 (1997) 338

[16] A. Retey and J. A. M. Vermaseren, Nucl. Phys. B 604 (2001) 281

[17] J. A. Gracey, Phys. Lett. B 322 (1994) 141; J. F. Bennett and J. A. Gracey, Nucl. Phys. B 517 (1998) 241

[18] S. Catani and F. Hautmann, Nucl. Phys. B 427 (1994) 475; V. S. Fadin and L. N. Lipatov, Phys. Lett. B 429 (1998) 127; M. Ciafaloni and G. Camici, Phys. Lett. B 430 (1998) 349

[19] J. Santiago, F.J. Yndurain, Nucl. Phys. B 611 (2001) 447

[20] W.L. van Neerven, A. Vogt, "Parton densities and structure functions at next-to-next-toleading order and beyond", 9th Intern. Workshop on Deep Inelastic Scattering (2001) Bologna, Italy, hep-ph/0107194

[21] A.L. Kataev, G. Parente, A.V. Sidorov, "Fixation of theor. ambiguities in the improved fits to $x F_{3}$ CCFR data at the NNLO order and beyond", hep-ph/0106221 (2001)

[22] CCFR/NuTeV Collab., U.K. Yang et al., Phys. Rev. Lett. 86 (2001) 2742

[23] D. Naples, "Structure functions from $\nu N$ scattering at Fermilab", presented at the Intern. Europhys. Conf. on High Energy Physics (2001), Budapest, Hungary

[24] E866/NuSea Collab., R.S. Towell et al., Phys. Rev. D 64 (2001) 052002

[25] CDF Collab., T. Affolder et al., Phys. Rev. D 64 (2001) 012001

[26] D0 Collab., B. Abbott et al., Phys. Rev. Lett. 86 (2001) 1707

[27] C. Pascaud, F. Zomer, H1 note h1-0295-426 (1995); V. Barone, C. Pascaud, F. Zomer, Eur. Phys. J C 12 (2000) 243

[28] M. Botje, Eur. Phys. J. C 14 (2000) 285; Nucl. Phys. Proc. Suppl. 79 (1999) 111

[29] W. T. Giele and S. Keller, Phys. Rev. D 58 (1998) 094023; hep-ph/0104053 (2001); W. T. Giele, S. A. Keller, and D. A. Kosower, hep-ph/0104052 (2001)

[30] J. Pumplin, D. Stump et al., "Uncertainties of predictions from parton distribution functions" hep-ph/0101051, hep-ph/0101032 (2001) 
[31] R.S. Thorne, A.D. Martin, W.James Stirling, R.G. Roberts, "MRST global fit update", 9th Intern. Workshop on Deep Inelastic Scattering (2001), Bologna, Italy, hep-ph/0106075

[32] W.K. Tung, private communications

[33] OPAL Collab., G. Abbiendi et al., Eur. Phys. J. C 18 (2000) 15

[34] L3 Collab., M. Acciarri et al., Phys. Lett. B 447 (1999) 147; 436 (1998) 403

[35] Pluto Collab., Ch. Berger et al., Nucl. Phys. B 2811987365

[36] R. Nisius, Phys. Rept. 332 (2000) 165

[37] M. Glück, E. Reya, I. Schienbein, Phys. Rev. D 64 (2001) 017501

[38] M. Krämer, S. Söldner-Rembold, Proc. of the Intern. Conf. on the Structure and Interactions of the Photon, and the 13th Intern. Workshop on Photon-Photon Collisions (2000), Ed. A.J. Finch, Ambleside, England, AIP Conference Proceedings 571 (2000)

[39] E. Witten, Nucl. Phys. B 120 (1977) 189

[40] H1 Collab., T. Ahmed, et al., Nucl. Phys. B 445 (1995) 195

[41] H1 Collab., C. Adloff et al., Phys. Lett. B 483 (2000) 36

[42] OPAL Collab., "Di-Jet Production in Photon-Photon Collisions at $\sqrt{s}$ from 189 to 202 GeV”, OPAL physics note PN443 (2000), abs. 176, XX Intern. Symp. on Lepton and Photon Interactions at High Energies (2001), Rome, Italy 